\title{
Etude de la variation de la végétation en milieux salé et acide au Sénégal
}

\author{
A. THIAM ${ }^{1 *}$, S.A.N. SAMBA ${ }^{2}$, K. NOBA ${ }^{3}$, J.P. NDIAYE ${ }^{4}$, M. DIATTA ${ }^{4}$ et M. WADE ${ }^{4}$ \\ ${ }^{I}$ Université de Thiès, Institut Supérieur de Formation Agricole et Rural (ISFAR), BP 54, Bambey, Sénégal. \\ ${ }^{2}$ Université de Thiès, Ecole Nationale Supérieure d'Agriculture (ENSA), BP 967, Thiès, Sénégal. \\ ${ }^{3}$ Université Cheikh Anta Diop (UCAD), Département de Biologie Végétale, BP 5005, Dakar, Sénégal. \\ ${ }^{4}$ Institut Sénégalais de Recherches Agricoles (ISRA), BP 2312, Dakar, Sénégal. \\ *Auteur correspondant, E-mail: amsathiam@yahoo.fr
}

\section{RESUME}

L'objectif de l'étude était de comprendre la variation de la végétation en milieux acide et salé. La répartition de la végétation des sols sulfatés acides salés en fonction de certains paramètres ( $\mathrm{pH}$, conductivité électrique ou CE, humidité du sol et texture des sols) a ainsi été étudiée dans les secteurs situés au Sud et au Nord du fleuve Saloum (Sénégal). Des relevés ont été effectués sur les différentes unités géomorphologiques (terrasses et dunes) suivant la méthode des grappes de superficies graduelles. Au niveau de chaque surface de collecte, des échantillons de sol ont été prélevés à $6 \mathrm{~cm}$ de profondeur pour déterminer leur $\mathrm{CE}, \mathrm{pH}$, teneur en eau et texture. L'étude a mis en évidence la distribution de la végétation en fonction des unités géomorphologiques et des types de faciès ainsi que les changements des faciès écologiques à travers les distributions aires-espèces. L'analyse en composantes principales a montré le rôle important de la texture du sol sur le regroupement de la végétation en fonction des gammes de salinité, d'acidité et du taux d'humidité. La connaissance du mode de regroupement de la végétation en milieux acide et salé pourrait aider dans le choix du type d'aménagement pour régénérer les potentialités agronomiques de ces sols et leur réintégration dans le système productif national.

(c) 2015 International Formulae Group. All rights reserved.

Mots clés: Acidité des sols, diversité biologique, humidité, salinité des sols, texture.

\section{INTRODUCTION}

Les terres salées et les sols sulfatés acides occupent plus d'un million d'hectares dans l'espace sénégambien. La salinisation des sols est un problème préoccupant pour leur mise en valeur agricole. Les tentatives de récupération des sols salés se sont très souvent confrontées à des problèmes imprévisibles de salinité excessive due à la faible pluviosité, à la sécheresse et aux phénomènes d'évaporation intense et de précipitation des sels en surface par remontées capillaires.
A l'arrière des mangroves s'étendent de vastes surfaces de sols généralement nues appelées localement tannes (wolof). Leur existence sur la plupart des littoraux à mangroves a été mise en évidence depuis longtemps (Guilcher, 1955; Berthois et Guilcher, 1956; Battistini, 1961; Fosberg, 1961; Lebigre et Marius 1984). La sursalure et l'acidification qui caractérisent les tannes ont façonné ces milieux entraînant des conséquences dramatiques sur l'environnement par la disparition de 70\% des 
forêts à Acacia et environ 30 à $40 \%$ des forêts à Combretum glutinosum (Sadio, 1986) et des systèmes de production (Thiam, 2007).

Les recherches menées depuis les années 60 ont montré qu'une voie d'utilisation possible de ces terres demeure la mise en valeur forestière par l'introduction d'espèces ligneuses halotolérantes. Cependant, à cause de la grande variabilité observée au niveau des propriétés de ces sols, une bonne caractérisation des milieux est nécessaire. C'est ainsi que nous nous sommes proposés d'étudier la variation de la végétation en fonction de la $\mathrm{CE}, \mathrm{du} \mathrm{pH}$ et de la texture des sols. Une telle approche a été faite d'une part par Diallo et al. (2009) sur la variabilité floristique de la végétation herbacée de la Niaye de Pikine (Sénégal) et d'autre part par Thiaw et al. (2011) sur la caractérisation de la végétation herbacée dans la région de Kaffrine (Sénégal).

L'étude de Diallo et al. (2009) a permis de caractériser les groupements végétaux tributaires des facteurs édaphiques et dont certains sont des vestiges d'un ancien tapis végétal et celle de Thiaw et al. (2011) a mis en évidence le regroupement des espèces végétales en fonction des paramètres physicochimiques du sol (texture du sol, bases échangeables, capacité d'échange cationique.).

La finalité de ce travail est de pouvoir identifier d'une part les gammes de salinité et d'acidité et d'autre part les textures des sols par le seul biais de la présence et du mode de regroupement de la végétation. L'étude permettra aussi la réduction des coûts d'analyses des échantillons des sols (granulométrie, conductivité et potentiel hydrogène) lors de la caractérisation de ces milieux.

\section{MATERIEL ET METHODES \\ Sites d'étude \\ Localisation}

L'étude a été menée au niveau du domaine des tannes $\left(14^{\circ} 08^{\prime}-14^{\circ} 20^{\prime}\right.$ latitude nord). Il s'étend plus au Nord jusqu'à la latitude $14^{\circ} 30^{\prime}$. Ce domaine, caractérisé par la présence des tannes, couvre plus des $2 / 3$ de la superficie totale du bassin. Deux sites ont été choisis pour mener cette étude: Ndiaffate $\left(14^{\circ}\right.$ 05'20 27' N; 16¹0'02 02', O) situé au Sud du fleuve Saloum et Ngan (14 ${ }^{\circ} 10^{\prime} 4311$ N', $16^{\circ} 05^{\prime} 5607^{\prime}$ ' O) situé au Nord du fleuve. Les sols sont de type sulfaté acide hydromorphe salé sur micro-dune sableuse à matériaux complexes (sables/argiles limoneuses).

\section{Le climat}

Le climat appartient au domaine sahélo-sénégalais (Leroux, 1990). Il est caractérisé par des températures moyennes mensuelles comprises entre 26 et $31{ }^{\circ} \mathrm{C}$ et par des précipitations normales de 600 à 900 mm/an (1931 - 1960) qui ne sont plus maintenant que de l'ordre de 400 à 600 $\mathrm{mm} /$ an (Leborgne, 1988). Le cumul annuel des précipitations (1985 à 2005) montre une variabilité zonale importante avec une moyenne pluviométrique de $596,8 \mathrm{~mm}$ et un écart-type de 113,4 mm. L'indice régional standardisé (IPS) caractérise une tendance globalement déficitaire (1985 à 2005).

A Ngan (Nord du fleuve), zone moins arrosée, la moyenne pluviométrique (1985 à 2005) est de 546,83 $\mathrm{mm}$ avec un écart type de 116,93 mm (Thiam, 2008) alors qu'à Ndiaffate (Sud du fleuve), zone plus arrosée, elle est de $668,4 \mathrm{~mm}$ pour un écart type de 154,24 mm (Thiam, 2008).

Unités géomorphologiques dans le domaine des tannes

Le domaine des tannes est constitué de différentes unités géomorphologiques: les tannes vasières, les terrasses basses inondables, les terrasses moyennes, les terrasses hautes, le glacis de raccordement, les dépressions et les dunes.

\section{Méthodes \\ Relevés de la végétation et du sol \\ Les différents types de faciès ou de tannes sur lesquels les relevés ont été effectués étaient: les tannes herbacées, les tannes localement arborées, tannes arborées et}


tannes arbustives. La méthode utilisée était celle des grappes de parcelles de superficies graduelles. Une grappe était constituée par un ensemble de superficies se succédant les unes aux autres et chaque surface considérée a été le double de la précédente. A partir d'une aire de $1 \mathrm{~m}^{2}$, la superficie a été doublée jusqu'à atteindre l'aire minimale de ce secteur, définie comme étant l'aire à partir de laquelle une augmentation de superficie n'apporte pas d'information significative en termes de nombre d'espèces (Braun Blanquet, 1928).

Pour chaque surface de collecte, des prélèvements d'échantillons de sol ont été effectués sur une tranche de sol de $6 \mathrm{~cm}$ de profondeur pour déterminer la conductivité électrique (CE) mesurée à l'aide d'un conductivimètre dans une solution sol/eau $(1 / 10)$ à $25^{\circ} \mathrm{C}$, le pH à l'aide d'un $\mathrm{pH}$-mètre à partir d'une solution sol/eau $(1 / 2,5)$, la teneur en eau par la méthode gravimétrique obtenue à partir du rapport :

$$
\mathrm{H}(\%)=\frac{\text { Poids frais }- \text { Poids sec }}{\text { Poids frais }} \times 100
$$

et la texture à partir du triangle texturale, après une analyse granulométrique pour la détermination des proportions de quatre fractions minérales du sol (sable fin, sable grossier, argile et limon).

\section{Analyse des données}

Le logiciel XLSTAT a été utilisé pour l'analyse en composante principale (ACP) dont l'objectif était de condenser l'information de manière à identifier les relations caractéristiques (proximités entre les variables et individus) tout en limitant la perte d'information. Weaver:

L'utilisation de l'indice de Shannon

$\boldsymbol{H}=\sum \boldsymbol{P}_{i} \log _{2} \boldsymbol{P}_{i}$

( $\mathrm{H}=$ indice de biodiversité de Shannon, $P_{i}$ la richesse spécifique $=n i / N$ avec $n i=$ nombre d'individus pour l'espèce $i$ et $N$ est l'effectif total) et du coefficient de similarité de Sorens:

$\mathrm{Ps}=(2 \mathrm{C} /(\mathrm{a}+\mathrm{b})) * 100$

$(\mathrm{a}=$ espèces du site de Ndiaffate $; \mathrm{b}$ celles de Ngan et c les espèces communes aux deux sites; ce coefficient varie de $0 \%$ à $100 \%$; Ps $=0$ s'il n'existe aucune espèce commune entre les deux sites et Ps $=100 \%$ quand les deux sites sont identiques) ont permis d'étudier la diversité et de faire la comparaison entre les communautés végétales de Ndiaffate et de Ngan.

\section{RESULTATS}

Distribution des tannes dans les terrasses moyennes et basses à Ndiaffate et Ngan

A Ngan, 50\% des tannes étaient localisés au niveau des terrasses moyennes contre $40 \%$ à Ndiaffate. Pour les terrasses basses inondables, on a enregistré $33 \%$ des tannes à Ngan et 30\% à Ndiaffate.

Etude de la végétation en fonction des unités géomorphologiques et du type de faciès

Ndiaffate, situé sur des sols sulfatés acides hydromorphes salés argilo-sableux à texture fine (taux d'argile > 24\%), a présenté une diversité biologique plus importante, avec un indice de Shannon Weaver de 3,28 (Tableau 2), que Ngan, moins arrosée et avec des sols sulfatés acides hydromorphes salés sur micro-dune sableuse à matériaux complexes (sables/argiles limoneuses) pour lesquels on a noté une texture grossière (taux de sable 55\%) et un indice de Shannon Weaver de 3,19 (Tableaux 1et 2).

La distribution du type de tannes, du nombre d'espèces et de genres n'a pas été fonction du type de terrasses au niveau des deux sites, de même qu'à l'intérieur d'un même site. Le coefficient de similarité de Sorensens $\mathrm{K}$ a été de $67 \%$ à Ndiaffate et de $68 \%$ à Ngan.

\section{Distribution aires / espèces dans les grappes à Ndiaffate et à Ngan Ndiaffate}

La distribution du type de tannes a varié en fonction du type de terrasse à Ndiaffate (Figure 1). La terrasse moyenne a présenté un faciès de tanne herbacée (Figure 
1B) alors que la terrasse basse a présenté un type de tanne localement arborée (Figure 1C).

Les changements de profils écologiques ont été observés à l'intérieur d'une même unité géomorphologique (terrasse basse) avec une succession de types de tannes (Figures 1C, 1D et 1F). Ce changement de profil écologique a aussi été observé lorsque le relevé prenait en compte deux unités géomorphologiques (terrasses moyennes et terrasses basses) contigües (Figures $1 \mathrm{E}$ et $1 \mathrm{G}$ ) avec respectivement la séquence: tannes arbustives > tannes nues > tannes localement arbustives.

Ngan

Le faciès tanne nue a été enregistré au niveau de la terrasse moyenne (Figure 2A) et les tannes herbacées au niveau des terrasses basses (Figures 2D et 2F); cependant, le gradient d'altitude entre les différentes unités géomorphologiques n'a pas été le seul facteur caractérisant la distribution des types de tannes (Figure 2B). En effet, la distribution spatio-temporelle très hétérogène des éléments de contraintes (salinité et acidité) a permis de mettre en évidence la diversité des faciès écologiques à l'intérieur d'une même unité géomorphologique ou entre deux unités géomorphologiques différentes se succédant. Le passage d'une unité à une autre a pu être mis en évidence par un changement du profil écologique (Figures 2C, 2E et 2G). Ce changement du profil a été observé lors du passage d'une terrasse moyenne vers une terrasse basse.

Distribution des familles par rapport aux grappes et aux dunes à Ndiaffate et à Ngan

A Ndiaffate, neuf (9) familles ont été les plus représentées au niveau des différentes grappes et des dunes. Les Poacées étaient présentes sur l'intégralité des grappes et des dunes, soit un pourcentage de $100 \%$. Cette représentativité a été de $50 \%$ pour les Rubiacées, $37,5 \%$ pour les Mimosacées et $25 \%$ pour les Malvacées. Les Papilionacées, Cypéracées, Césalpiniacées, Fabacées et
Asclépiadacées étaient représentées chacune à hauteur de $12,5 \%$.

A Ngan, les Poacées et les Rubiacées étaient présentes sur la totalité des relevés avec un pourcentage de $100 \%$. En revanche les Mimosacées et les Malvacées étaient présentes sur $25 \%$ des relevés alors que les Tiliacées et les Fabacées n'étaient présentes que dans $12,5 \%$ des relevés.

\section{Distribution des espèces en fonction des propriétés du sol \\ Ndiaffate}

Les espèces recensées étaient présentes sur trois types de texture: sablo limoneuse, limono - sableuse et sableuse. L'analyse en composante principale (ACP) a permis de mettre en évidence différents groupes en fonction des textures du sol. Pour la texture sableuse (Figure 3), les axes F1 et F2 ont expliqué $93 \%$ de la variabilité totale entre variables et individus. L'axe F1 a expliqué à lui seul 63\% et l'axe F2 30\%. Pour le pourcentage des contributions des variables par rapport aux axes, l'humidité a contribué pour $47 \%$ pour l'axe $\mathrm{F} 1$ et la conductivité électrique pour $81 \%$ pour l'axe F2.

Avec les individus, Chloris pilosa a contribué pour $35 \%$ pour l'axe F1 et Dichostachys glomerata pour $83 \%$ pour l'axe F2. Avec l'utilisation des deux axes F1 x F2, trois groupes se sont distingués, le groupe 1 constitué par D. glomerata seul, le second groupe composé par Leptadenia hastata, Phyloxerus vermicularis, C. pilosa, Digitaria ciliaris et Merremia tridentata.

L'utilisation du pourcentage des contributions des individus a permis de noter à Ndiaffate, pour les textures sableuses, que $D$. glomerata caractérisaient les sols très salins $(8$ $\mathrm{dS} / \mathrm{m}$ ) avec un taux d'humidité de $2 \%$. Les sols légèrement et moyennement salins]0,41 $1,07 \mathrm{dS} / \mathrm{m}$ ] dont le taux d'humidité variait de $0,23 \%$ à $1,39 \%$ étaient caractérisés par $M$. tridentata. C. pilosa caractérisait les sols légèrement salins à salins ]2,5 - 4,05 ds/m] avec des teneurs d'humidité de 2 à $5 \%$. 
Pour la texture sablo-limoneuse (Figure 4), les axes F1 et F2 ont expliqué $84 \%$ de la variabilité entre variables et individus (F1: 46\%; F2: 38\%). Pour la contribution des variables, le $\mathrm{pH}$ a contribué pour $54 \%$ par rapport à $\mathrm{F} 1$ et la conductivité électrique pour $75 \%$ par rapport à F2.

Avec les pourcentages de contribution des individus, Oryza longistaminata a contribué pour $24 \%$ pour l'axe $\mathrm{F} 1$ et $D$. glomerata pour $36 \%$ pour l'axe F2. Avec ces deux axes, quatre groupes se sont distingués. Le groupe 1 était constitué par Oryza longistaminata, Conocarpus erectus, Sphaeranthus senegalensis, Chloris gayana, Cyperus rotundus. Le second était constitué de D. glomerata, Indigofera tintoria, Acacia nilotica, Leptadenia hastata, Commiphora africana. Le troisième groupe contenait Acanthospermum hispidum, Ctenium elegans, Spermacoce verticillata, Eragrostis squamata, Corchorus olitorius, Balanites aegyptiaca, Sida alba, Cassia obtusifolia. Le groupe quatre comprenait Spermacoce verticillata, Dactyloctenium aegyptium, Eragrostis tremula, Kohautia grandiflora. Ces groupes étaient opposés deux à deux, le groupe 1 au groupe 3 et le groupe 2 au groupe 4 .

Les informations obtenues des pourcentages des contributions des espèces par rapport aux deux axes lors de l'ACP ont mis en évidence les quatre espèces les mieux appropriées pour la caractérisation de ces sols à texture sablo-limoneuse: $C$. erectus, $D$. glomerata, B. aegyptiaca et Kohautia grandifolia. C. erectus caractérisait les sols salins ]2,86 - 4,33 dS/m] avec des acidités variant de très acide à acide]4,2 - 5,5]. $D$. glomerata se retrouvait au niveau des sols salins à très salins ]3,27 à $8,22 \mathrm{dS} / \mathrm{m}]$ pour une faible acidité]5,28 - 6,03]. B. aegyptiaca caractérisait les sols moyennement salins à salins ]1,28 - 3,08 dS/] pour des acidités variant d'acide à faiblement acide]5,28 6,03]. K. grandifolia indiquait les sols moyennement salins à salins ]1,29 - 2,99 $\mathrm{dS} / \mathrm{m}]$ pour des acidités variant de très acide à faiblement acide]4,34 - 5,56].
Pour la texture limono-sableuse (Figure 5), les axes $\mathrm{F} 1$ et $\mathrm{F} 2$ ont expliqué $83 \%$ de la variabilité entre individus et variables (F1: 49\%; F2: 34\%). Pour les variables, le pH a contribué pour $50 \%$ par rapport à $\mathrm{F} 1$ et la conductivité électrique $95 \%$ pour $\mathrm{F} 2$.

$P$. aculeata, N. lotus et A. gayanus contribuaient respectivement pour 40,17 et $15 \%$, soit un cumul de $72 \%$, par rapport à l'axe 1. Pour l'axe 2, Acacia nilotica a contribué pour $10 \%$. L'utilisation des deux axes $\mathrm{F} 1$ et $\mathrm{F} 2$ a fait ressortir quatre groupes distincts. Le premier groupe était constitué par Tamarix senegalensis, $P$. aculeata, $S$. senegalensis, Hibiscus asper, Brachiaria ramosa, Combretum glutinosum et Spermacoce stachydea. A. nilotica, Cyperus rotundus, Mytragyna inermis, Acacia seyal, Commiphora africana, Dactyloctenium aegyptium, Zornia glochidiata, Cassia obtusifolia, Sida alba, Enterpogon prieurii, Sida rhombifolia, Acanthospermum hispidum et Ctenium elegans étaient associées au second groupe. Le troisième groupe était composé parSpermacoce verticillata, Eragrostis squamata, Corchorus olitorius, A. gayanus, Pycreus albomarginatus, Azadiracta indica, Ziziphus mauritiana, Piliostigma reticulatum, Pennisetum pedicellatum, Ipomaea aquatica, Haemanthus pentaphylla et Alysicarpus ovalifolius. Le quatrième groupe regroupait $N$. lotus, Crotolaria arenaria et Calotropis procera.

Les valeurs des pourcentages des contributions par rapport aux axes mettaient en évidence que dans ces sols sulfatés acides salés à texture limono-sableuse pour des gammes de salinités et d'acidités variables, $P$. aculeata, A. nilotica, A. gayanus et $N$. lotus caractérisaient mieux ces sols. $P$. aculeata caractérisait les sols moyennement salins à salins ]1,43 - 2,96 dS/m] avec des gammes d'acidités de très acide à acide ]4,35 - 5,05]. Pour cette même texture, $A$. nilotica caractérisait les sols salins ]2,38 - 4,27 dS/m] avec des caractères d'acidité variant d'acide à faiblement acide 15,18 - 6,19]. A. gayanus et 
$N$. lotus caractérisaient les sols non salins à moyennement salins ]0,06 - 2,04dS/m].

Ngan

Pour la texture sableuse (Figure 6), les axes $\mathrm{F} 1$ et $\mathrm{F} 2$ expliquaient $93 \%$ de la variabilité (F1: 68\%; F2: 25\%). Avec le pourcentage de contribution des variables, l'humidité a participé à hauteur de $40 \%$ pour l'axe $\mathrm{F} 1$ et le $\mathrm{pH}$ à $80 \%$ pour l'axe F2. Avec les individus, B. aegyptiaca a contribué pour $19 \%$ pour l'axe $\mathrm{F} 1$ et $H$. asper $45 \%$ pour l'axe F2. L'utilisation à la fois des deux axes fait ressortir quatre groupes distincts. Le premier renfermait Cenchrus biflorus, C. pilosa, Cynodon dactylon, Acacia albida et Schoenefeldia gracilis. Le second regroupait Ctenium elegans, S. verticillata, Enteropogon preurii, Cyperus rotundus, Cassia obstusifolia et $B$. aegyptiaca. Le troisième était constitué par Dactyloctenium aegyptium, Ampelocisus pentaphylla, Sida rhombifolia, Zornia glochidiata et Corchorus olitorius. C. procera, Ocimum basilicum, Philoxerus vermicularis, Waltheria indica, Eragrostis tenella, et Hibiscus asper étaient associées au groupe 4 .

L'utilisation des pourcentages des contributions des individus a mis en évidence que $C$. biflorus, $C$. obstusifolia, $C$. olitorius et $H$. asper caractérisaient les sols sulfatés acides salés situés à Ngan et présentant une texture sableuse pour des gammes d'acidité et de salinité variables. C. biflorus et $H$. asper caractérisaient ces sols variant de non salin à moyennement salin ]0,03 - 1,69 dS/m] pour des gammes d'acidité variant d'acide à neutre ]4,48 - 6,8]. C. obstusifolia a caractérisé les sols moyennement salins à salins ]1,7 - 3,37 $\mathrm{dS} / \mathrm{m}]$, acides à faiblement acides [5,22 5,75]. Cassia obstusifolia caractérisait les sols moyennement salins ]1,44 - 2,26 ds/m] et acides ]5,14 - 5,6].

Avec la texture sablo-limoneuse (Figure 7), les résultats de l'ACP ont montré que les axes F1 et F2 expliquaient la quasitotalité de la variabilité $(98 \%)$ avec respectivement $84 \%$ pour $\mathrm{F} 1$ et $14 \%$ pour F2. Pour l'utilisation des pourcentages des contributions, l'humidité du sol a participé à hauteur de $37 \%$ par rapport à l'axe $\mathrm{F} 1$ et le $\mathrm{pH}$ pour $69 \%$ pour l'axe F2. Avec les individus, Sesuvium portulacastrum a eu la plus importante contribution (47\%) pour l'axe F1 et Cenchrus biflorus la plus importante (25\%) pour l'axe F2.

Quatre groupes ont été répertoriés avec l'utilisation des deux axes: le groupe 1 opposé au groupe 3 et le groupe 2 opposé au groupe 4. Les espèces du groupe 1 étaient $C$. biflorus, C. pilosa, C. elegans, A. nilotica et $S$. gracilis. Le second regroupait $S$. verticillata, $B$. aegyptiaca, A. seyal et $S$. portulacastrum. Le groupe 3 renfermait Andropogon pseudapricus, C. rotundus, E. preurii, $S$. stachydea, S. rhombifolia, Ampelocisus pentaphylla et Z. glochidiata et le groupe 4: D. aegyptium, E. squamata, Peristrophe bicalyculata, Philoxerus vermicularis, $E$. tenella, Thypha australis et E. tremula.

Avec les pourcentages des contributions des individus, C. biflorus, $S$. portulacastrum, Z. glochidiata, E. tremula étaient les plus appropriées pour caractériser ces sols à texture sablo-limoneuse. C. biflorus caractérisait les sols faiblement acides ]5,71 6,89 ] dont la teneur d'humidité variait de 0,2 à $2,86 \%$. Pour une gamme d'acidités variant de très acide à faiblement acide ]4,48 - 5,75], Sesuvium portulacastrum constituait un bon indicateur lorsque la teneur en eau variait entre 2,73 et $8,98 \%$. Z. glochidiata caractérisait ces sols lorsque leur $\mathrm{pH}$ variait entre 5,14 et 5,52 et leur humidité entre 1,45 à $3,49 \%$. Pour les sols acides à faiblement acides ]5,15 - 5,86] à humidité variant de 0,99 à 2,44\%, Eragrostis tremula était la mieux appropriée pour caractériser ces sols.

Avec la texture limono-sableuse (Figure 8), les relations entre individus et variables ont été expliquées à 95\% par les axes F1 (59\%) et F2 (36\%). Dans l'ACP, l'axe F1 a été expliqué par la conductivité électrique $(51 \%)$ et l'humidité (49\%). Pour l'axe F2, le pH a contribué pour $91 \%$.

L'utilisation des pourcentages des contributions des individus a montré que $A$. 
pseudapricus a contribué à hauteur de $48 \%$ pour l'axe F1 et Commelina forskalaei à hauteur de $22 \%$ pour l'axe F2. La combinaison des deux axes a fait ressortir quatre groupes distincts qui s'opposent deux à deux. Le groupe 1 était constitué par $C$. forskalaei, S. dulcis et E. squamata. Le groupe 2 regroupait $S$. senegalensis, $K$. grandiflora et I. asarifolia. Le groupe 3 était composé par Brachiaria ramosa, Peristrophe bicalyculata et A. pseudapricus. Les individus associés au groupe 4 étaient Crotalaria glaucoïdes, Anarcadium occidentalis, Pennisetum pedicellatum et Cassia nigricans.

L'utilisation des pourcentages des contributions des individus par rapport aux axes a permis de mettre en évidence que les espèces $C$. forskalaei, $K$. grandiflora, $A$. pseudapricus, ainsi que le groupe d'espèces C. glaucoïdes, A. occidentalis et $P$. pedicellatum caractérisaient mieux les sols sulfatés acides salés situés au nord du fleuve Saloum (Ngan) et dont la texture est limonosableuse. C. forskalaei, K. grandiflora et le groupe constitué parC. glaucoüdes, $A$. occidentalis et $P$. pedicellatum caractérisaient ces sols non salins à moyennement salins ]0,01-0,76 dS/m] acide à faiblement acide ]5,41 - 6,22]. A. pseudapricus indiquait les sols légèrement salins à salins ] 0,67 - 2,3 $\mathrm{dS} / \mathrm{m}]$, acide à faiblement acide ]5,35 - 5,68].

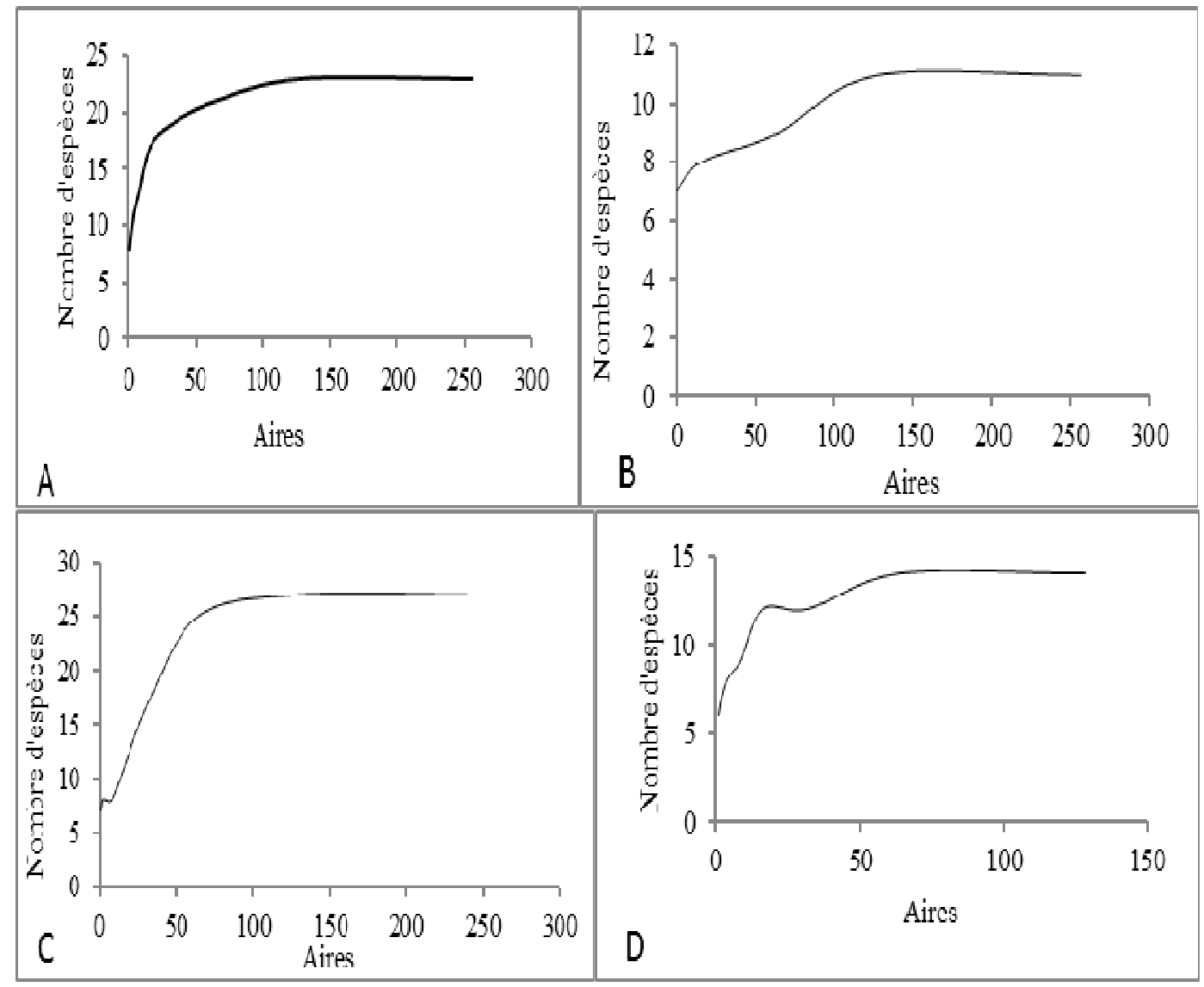




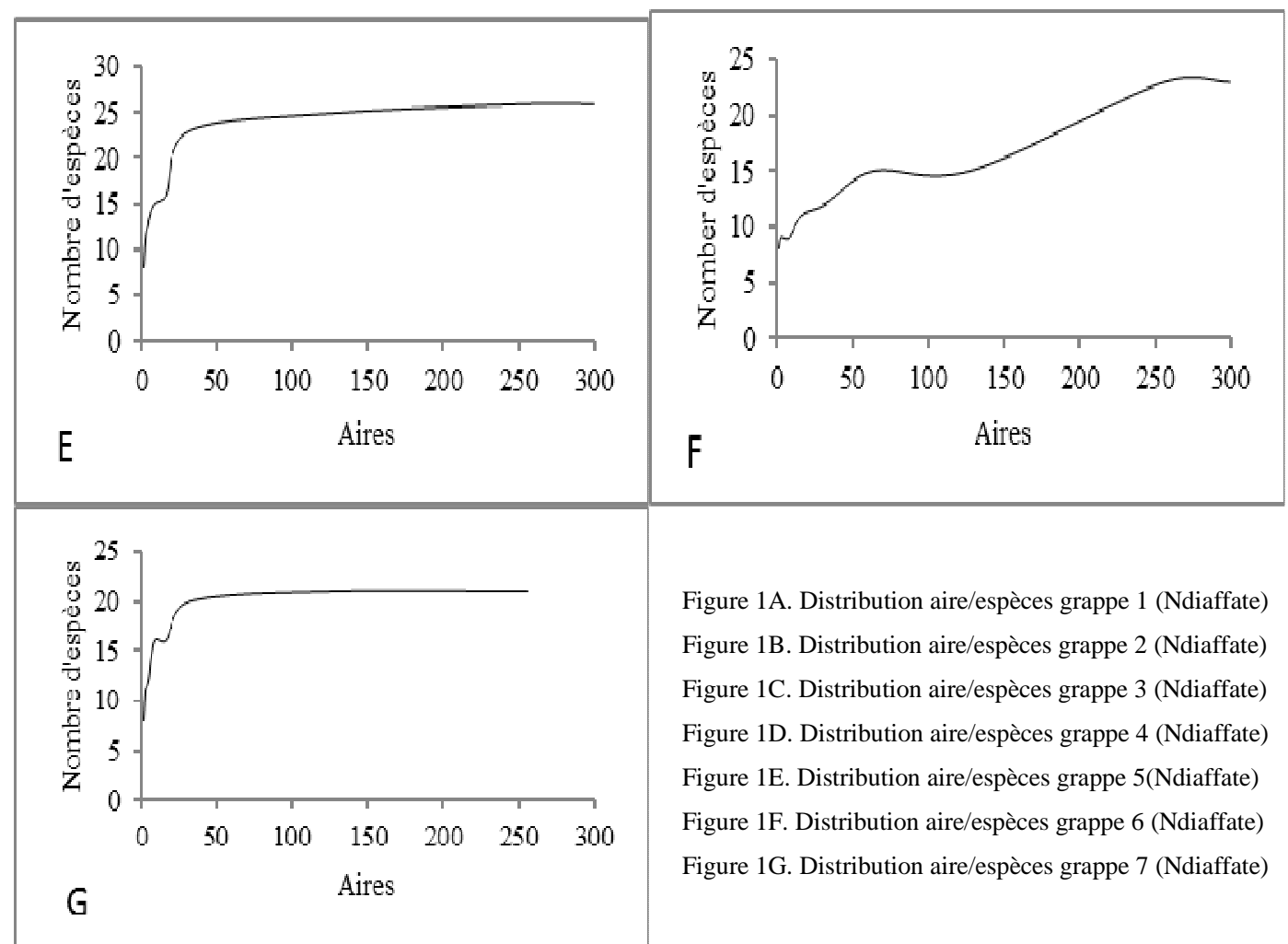

Figure 1. Distribution aire /espèces dans les différentes grappes (Ndiaffate).

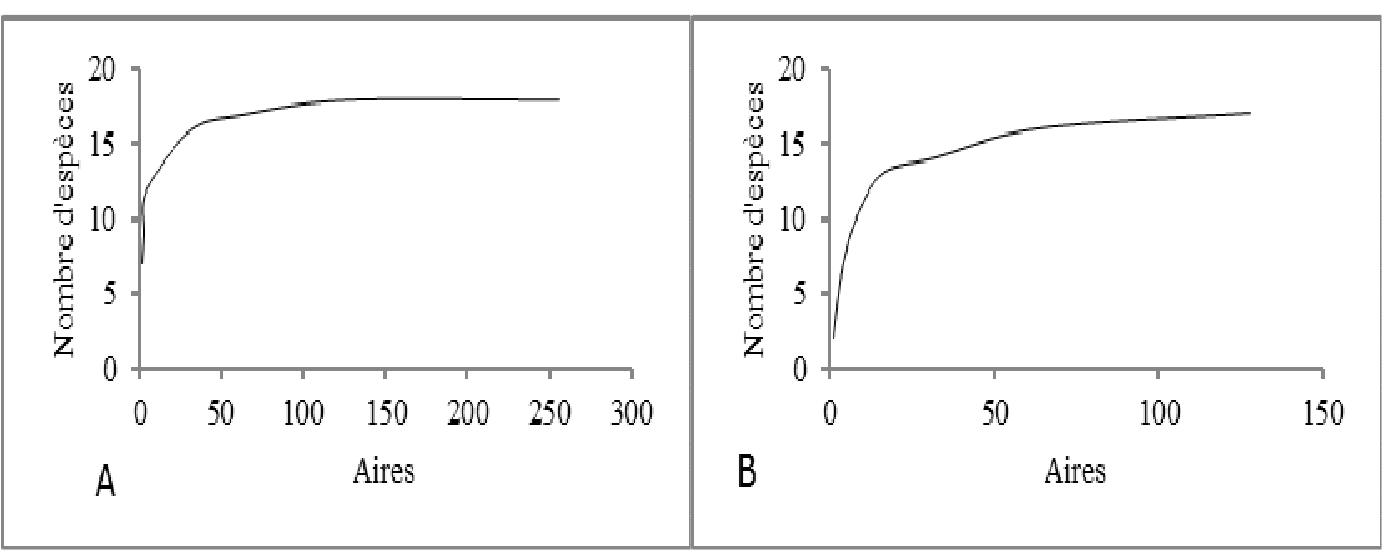


A. THIAM et al. / Int. J. Biol. Chem. Sci. 9(1): 155-175, 2015
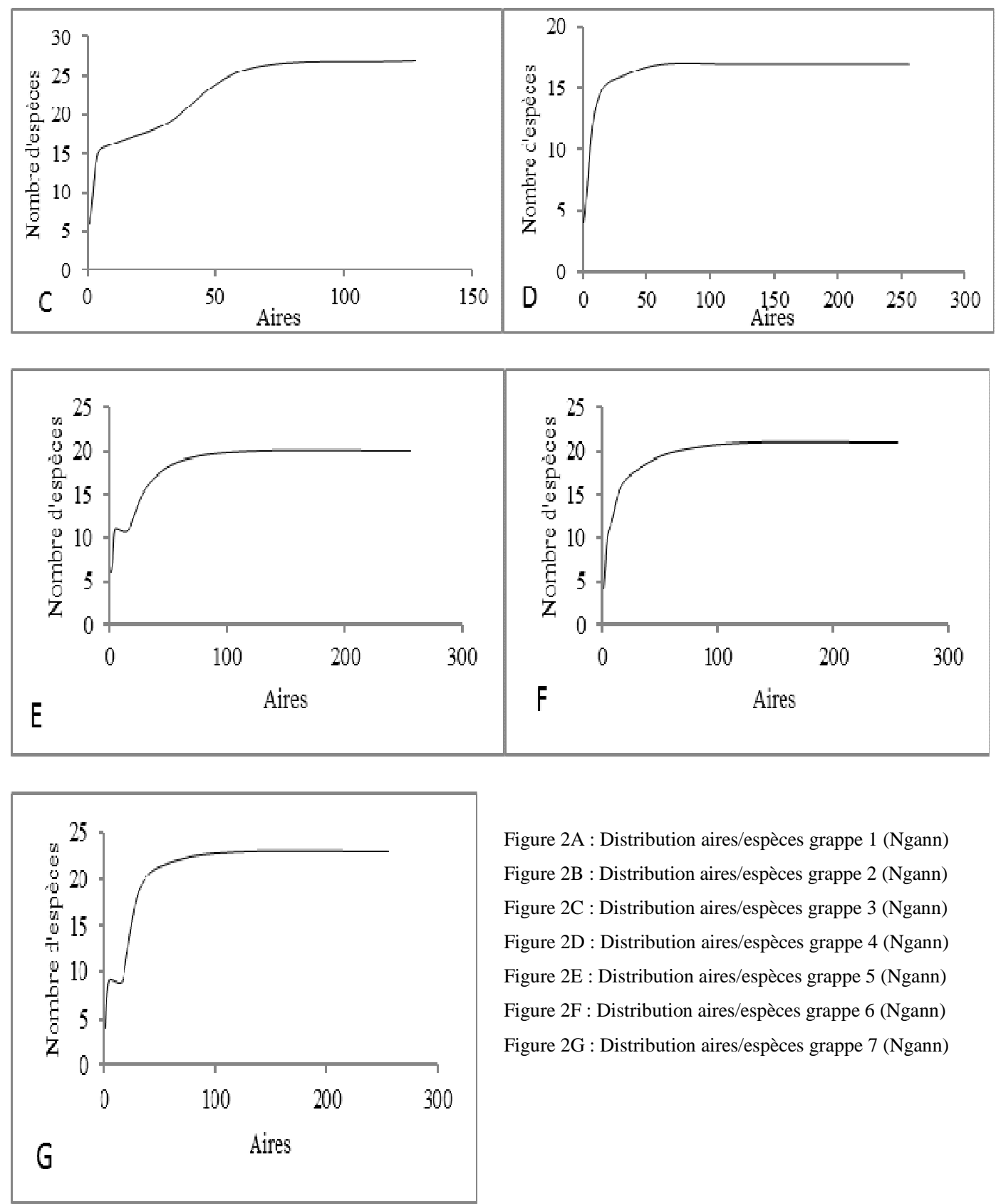

Figure 2A : Distribution aires/espèces grappe 1 (Ngann) Figure 2B : Distribution aires/espèces grappe 2 (Ngann) Figure 2C : Distribution aires/espèces grappe 3 (Ngann) Figure 2D : Distribution aires/espèces grappe 4 (Ngann) Figure 2E : Distribution aires/espèces grappe 5 (Ngann) Figure 2F : Distribution aires/espèces grappe 6 (Ngann) Figure 2G : Distribution aires/espèces grappe 7 (Ngann)

Figure 2 : Distribution aire /espèces dans les différentes grappes (Ngan). 


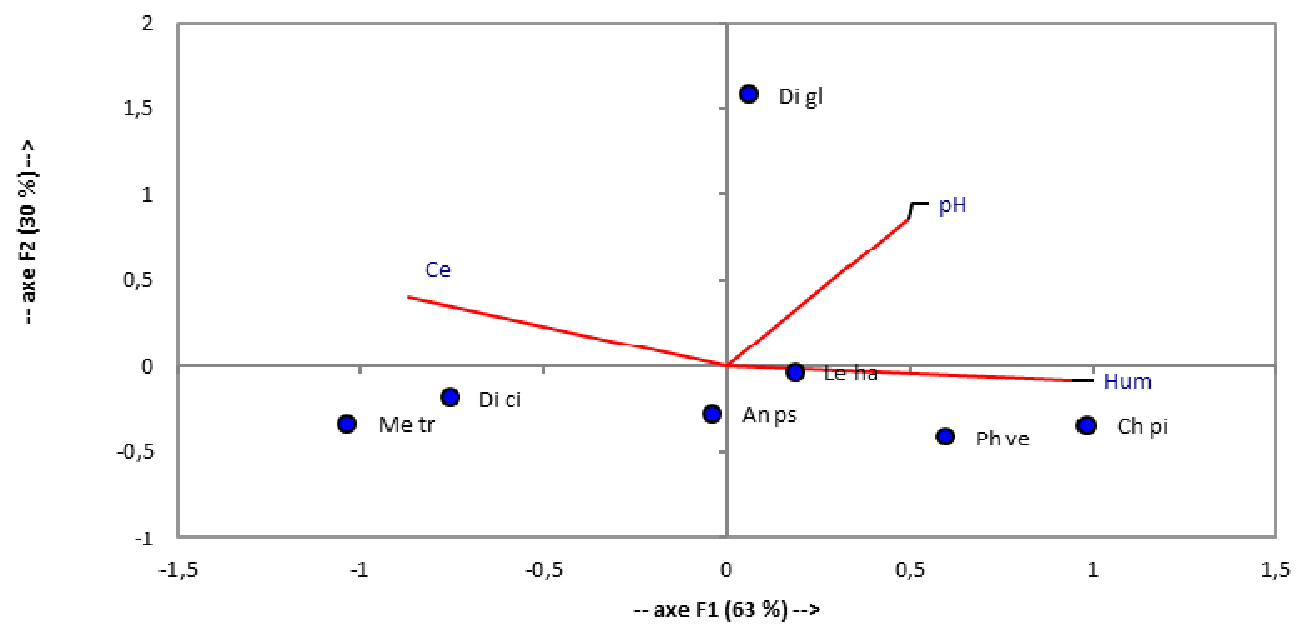

Figure 3 : Distribution des espèces en fonction de la texture sableuse, de la conductivité électrique (Ce dS/m), du pH et du taux d'humidité (Hum) (Ndiaffate).

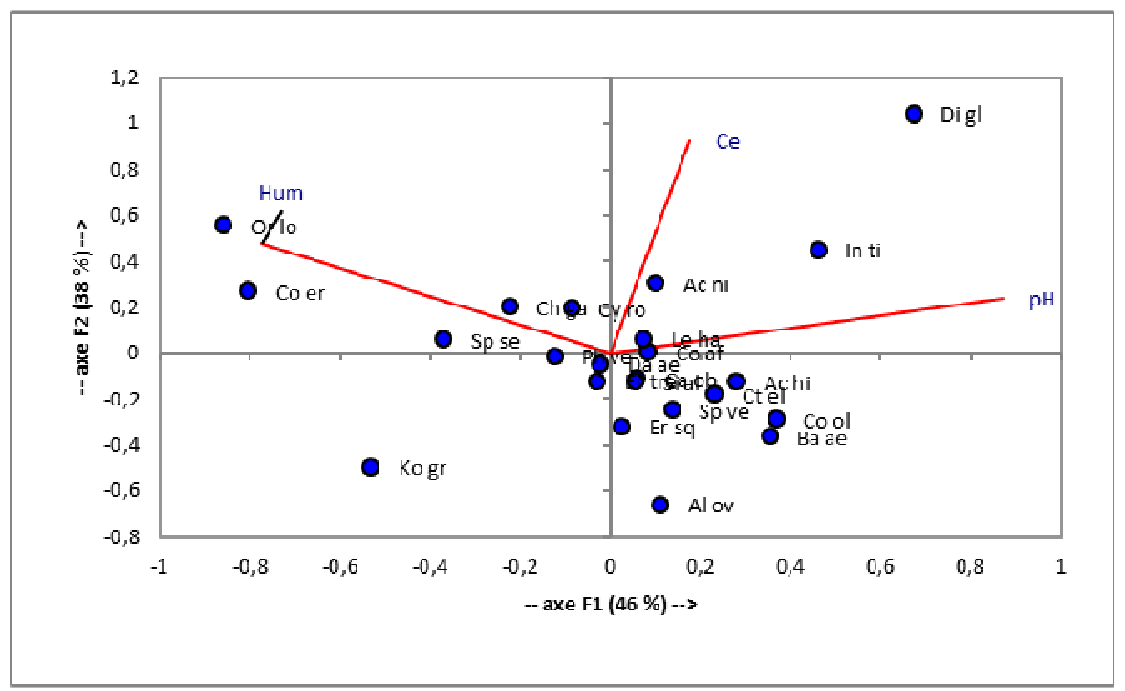

Figure 4 : Distribution des espèces en fonction de la texture sablo-limoneuse, de la conductivité électrique (Ce dS/m), du pH et du taux d'humidité (Hum) (Ndiaffate). 


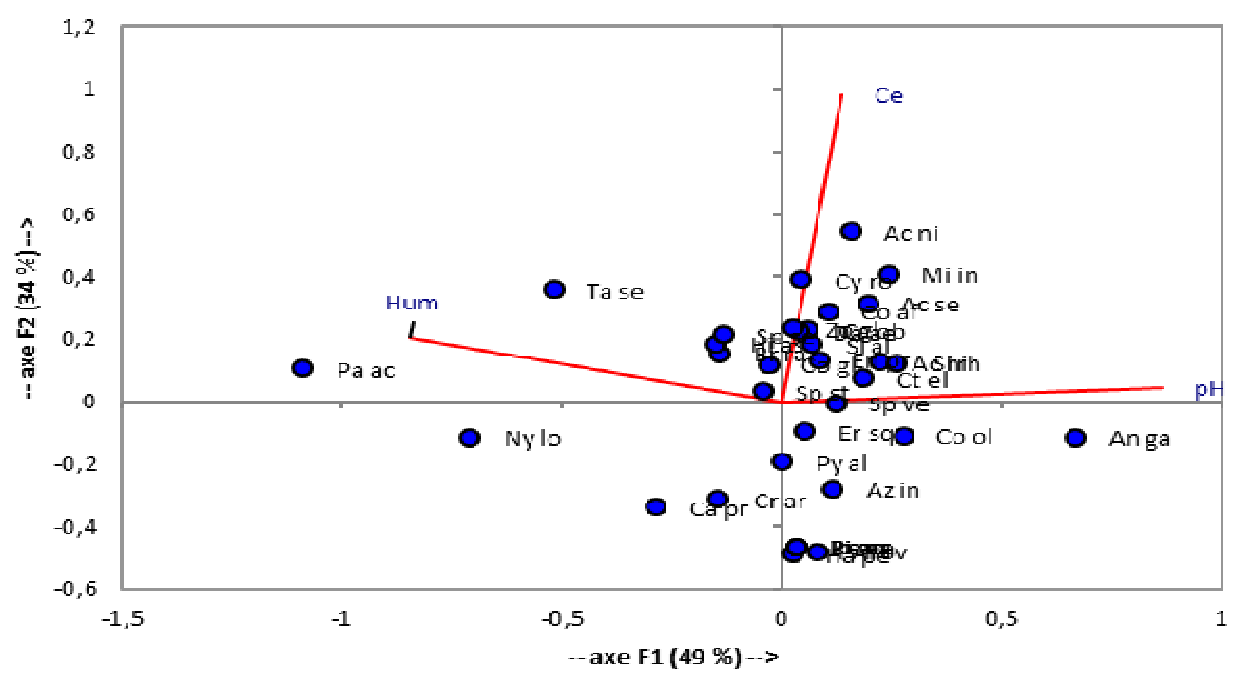

Figure 5: Distribution des espèces en fonction de la texture limono-sableuse, de la conductivité électrique (Ce dS/m), du pH et du taux d'humidité (Hum) (Ndiaffate).

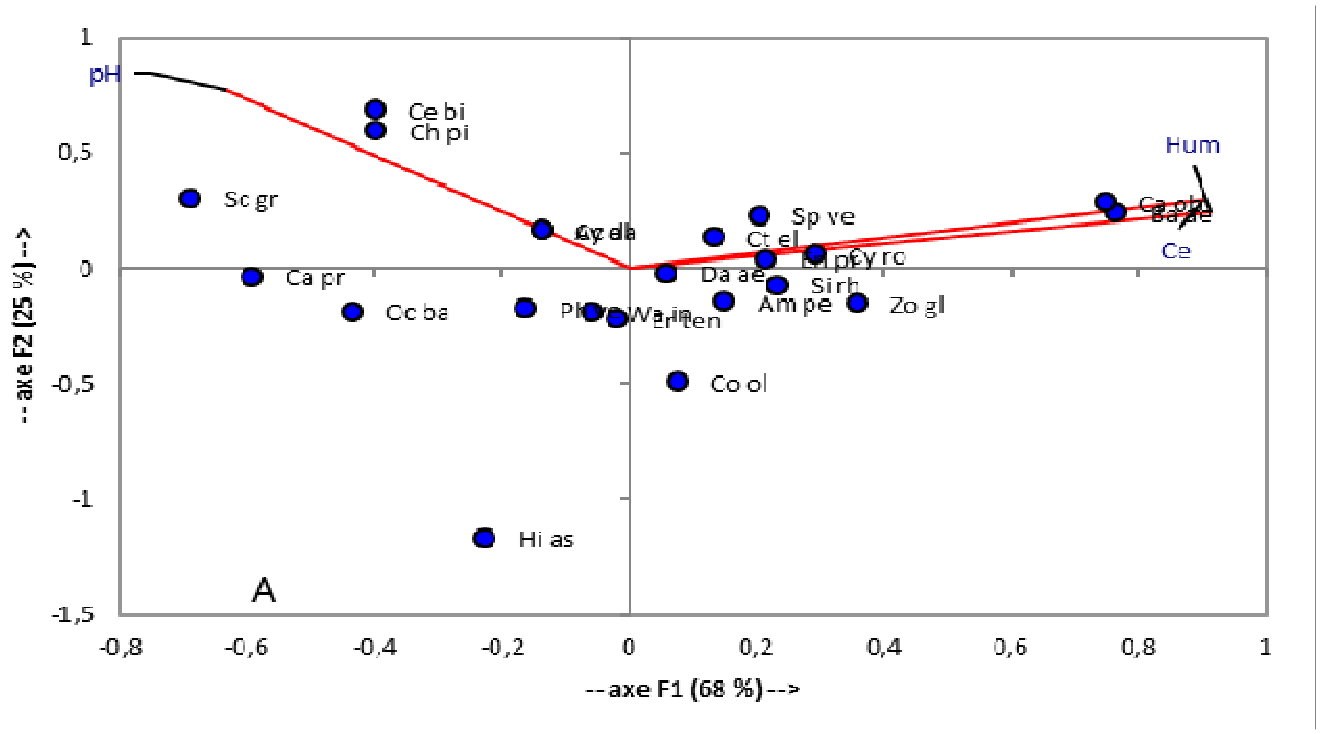

Figure 6 : Distribution des espèces en fonction de la texture sableuse, de la conductivité électrique (Ce, dS/m), du pH et du taux d'humidité (Hum) (Ngan). 


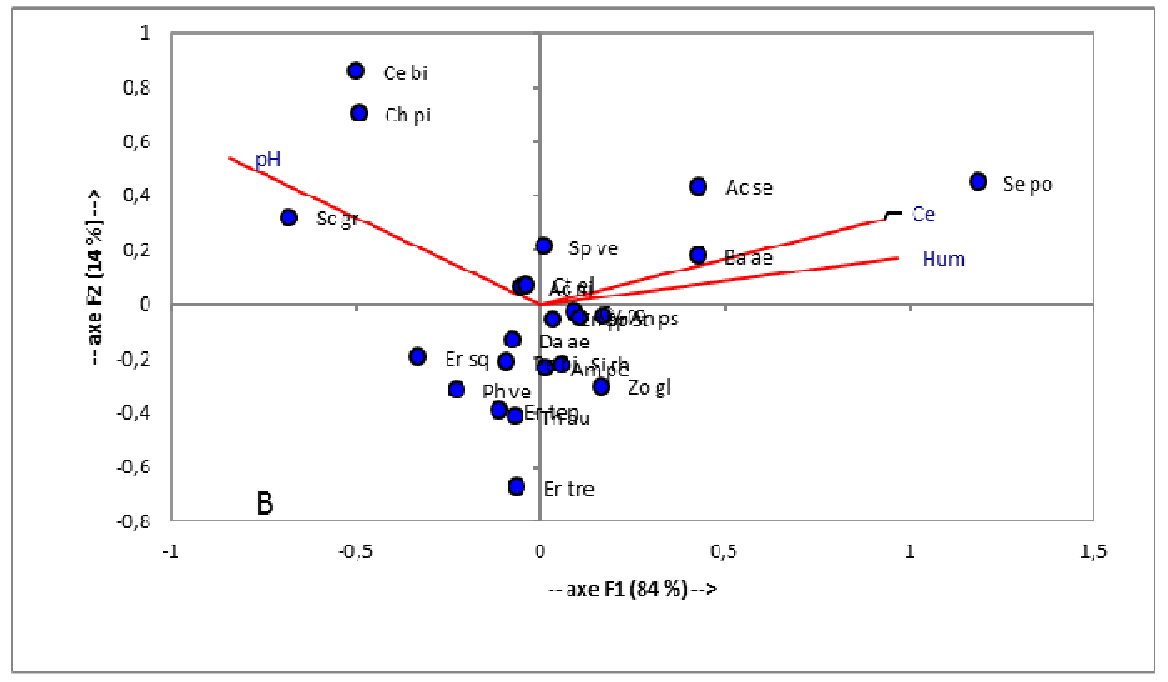

Figure 7 : Distribution des espèces en fonction de la texture sablo-limoneuse, de la conductivité électrique (Ce dS/m), du pH et du taux d'humidité (Hum) (Ngan).

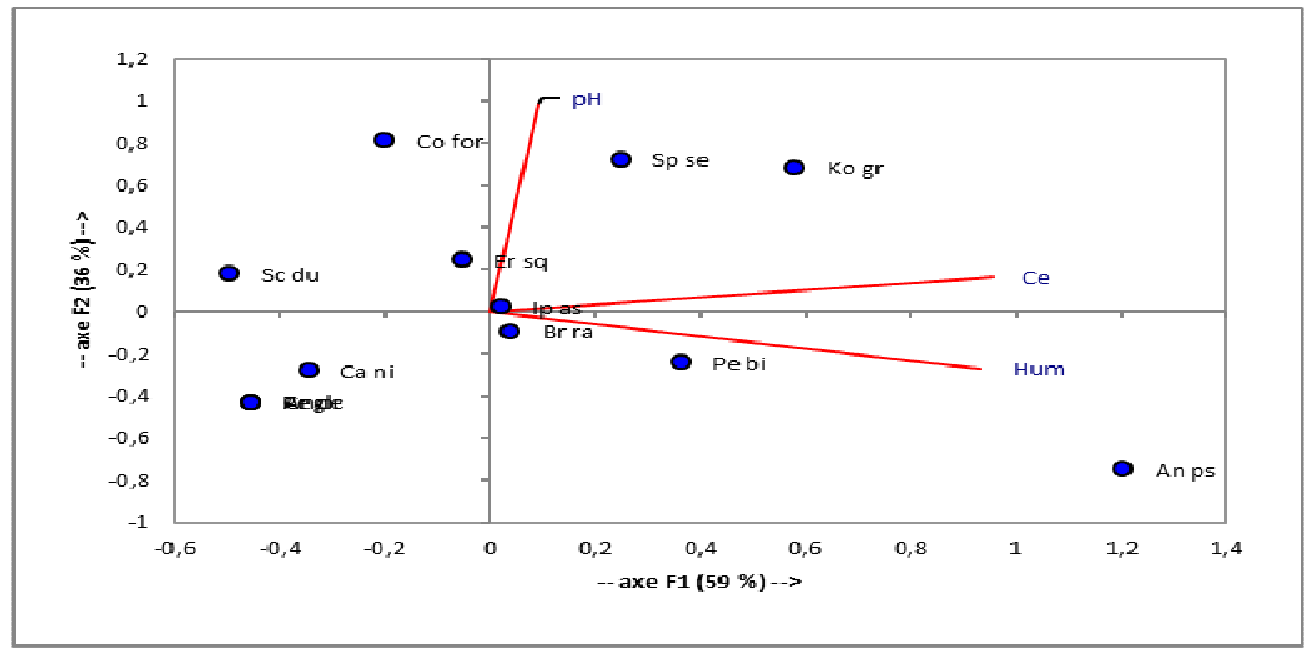

Figure 8. Distribution des espèces en fonction de la texture limono-sableuse, de la conductivité électrique (Ce dS/m), du pH et du taux d'humidité (Hum) (Ngan). 
A. THIAM et al. / Int. J. Biol. Chem. Sci. 9(1): 155-175, 2015

Tableau 1 : Données floristiques du site de Ndiaffate.

\begin{tabular}{|c|c|c|c|c|c|c|c|c|c|}
\hline Espèces & $\mathbf{F}$ & $\mathbf{P}$ & CS\% & FS\% & $\mathbf{P i}$ & $\mathbf{P i} \mathbf{L} \mathbf{L}(\mathbf{P i})$ & $\mathbf{p H}$ & $\mathrm{Ce}$ & Hum \\
\hline Philoxerus vermicularis & Amaranthacée & 61 & 11,07 & 82,43 & 0,11 & $-0,24$ & 5,7 & 5,0 & 15,3 \\
\hline Cyperus rotundus & Cypéracée & 45 & 8,17 & 60,81 & 0,08 & $-0,20$ & 5,7 & 5,0 & 15,3 \\
\hline Dactyloctenium aegyptiacum & Paocée & 40 & 7,26 & 54,05 & 0,07 & $-0,19$ & 5,7 & 5,0 & 3,9 \\
\hline Enteropogon prieurii & Poacée & 35 & 6,35 & 47,30 & 0,06 & $-0,18$ & 5,8 & 5,0 & 3,7 \\
\hline Eragrostis tremula & Poacée & 34 & 6,17 & 45,95 & 0,06 & $-0,17$ & 5,7 & 3,8 & 4,0 \\
\hline Sphaeranthus Senegalensis & Astéracée & 33 & 5,99 & 44,59 & 0,06 & $-0,17$ & 5,3 & 5,0 & 15,5 \\
\hline Ctenium elegans & Poacée & 26 & 4,72 & 35,14 & 0,05 & $-0,14$ & 5,5 & 5,0 & 3,8 \\
\hline Spermacoce verticillata & Rubiacée & 25 & 4,54 & 33,78 & 0,05 & $-0,14$ & 5,6 & 3,7 & 3,6 \\
\hline Cassia obtusifolia & Césalpinacée & 24 & 4,36 & 32,43 & 0,04 & $-0,14$ & 5,0 & 5,0 & 2,3 \\
\hline Zornia glochidata & Fabacée & 22 & 3,99 & 29,73 & 0,04 & $-0,13$ & 5,1 & 5,0 & 2,2 \\
\hline Eragrostis squamata & Poacée & 20 & 3,63 & 27,03 & 0,04 & $-0,12$ & 5,7 & 3,4 & 5,7 \\
\hline Balanites aegyptiaca & Simaroubacée & 16 & 2,90 & 21,62 & 0,03 & $-0,10$ & 5,9 & 2,6 & 1,6 \\
\hline Combretum glutinosum & Combretacée & 16 & 2,90 & 21,62 & 0,03 & $-0,10$ & 5,5 & 5,0 & 3,9 \\
\hline Andropogon pseudapricus & Paocée & 13 & 2,36 & 17,57 & 0,02 & $-0,09$ & 5,2 & 5,0 & 3,8 \\
\hline Leptadenia hastata & Asclépiadacée & 12 & 2,18 & 16,22 & 0,02 & $-0,08$ & 5,5 & 3,7 & 3,8 \\
\hline Acacia nilotica & Mimosacée & 11 & 2,00 & 14,86 & 0,02 & $-0,08$ & 5,7 & 4,1 & 5,1 \\
\hline Oryza longistaminata & Poacée & 11 & 2,00 & 14,86 & 0,02 & $-0,08$ & 5,7 & 3,5 & 16,1 \\
\hline
\end{tabular}


A. THIAM et al. / Int. J. Biol. Chem. Sci. 9(1): 155-175, 2015

\begin{tabular}{|c|c|c|c|c|c|c|c|c|c|}
\hline Acacia seyal & Mimosacée & 10 & 1,81 & 13,51 & 0,02 & $-0,07$ & 5,6 & 5,0 & 3,5 \\
\hline Sida alba & Malvacée & 10 & 1,81 & 13,51 & 0,02 & $-0,07$ & 5,5 & 2,9 & 1,7 \\
\hline Chloris pilosa & Poacée & 9 & 1,63 & 12,16 & 0,02 & $-0,07$ & 5,7 & 5,3 & 4,1 \\
\hline Mitragyna inermis & Rubiacée & 9 & 1,63 & 12,16 & 0,02 & $-0,07$ & 5,6 & 4,1 & 4,1 \\
\hline Spermacoce stachydea & Rubiacée & 8 & 1,45 & 10,81 & 0,01 & $-0,06$ & 5,0 & 2,9 & 1,7 \\
\hline Commiphora africana & Buséracée & 6 & 1,09 & 8,11 & 0,01 & $-0,05$ & 5,5 & 5,0 & 3,9 \\
\hline Brachiaria ramosa & Poacée & 5 & 0,91 & 6,76 & 0,01 & $-0,04$ & 4,7 & 5,0 & 3,6 \\
\hline Azadirachta indica & Méliacée & 4 & 0,73 & 5,41 & 0,01 & $-0,04$ & 5,6 & 2,6 & 1,6 \\
\hline Corchorus olitorius & Tiliacée & 4 & 0,73 & 5,41 & 0,01 & $-0,04$ & 6,0 & 2,6 & 1,7 \\
\hline Haemanthus pentaphylla & Amarylldacée & 4 & 0,73 & 5,41 & 0,01 & $-0,04$ & 5,2 & 0,1 & 0,7 \\
\hline Pycreus albomarginatus & Cypéracée & 4 & 0,73 & 5,41 & 0,01 & $-0,04$ & 5,5 & 1,2 & 3,8 \\
\hline Tamarix senegalensis & Tamaricacée & 4 & 0,73 & 5,41 & 0,01 & $-0,04$ & 5,7 & 4,1 & 16,7 \\
\hline Calotropis procera & Asclépiadacée & 3 & 0,54 & 4,05 & 0,01 & $-0,03$ & 4,5 & 0,7 & 4,6 \\
\hline Dichrostachys glomerata & Mimosacée & 3 & 0,54 & 4,05 & 0,01 & $-0,03$ & 6,0 & 8,7 & 2,9 \\
\hline Acanthospermum hispidum & Astéracée & 2 & 0,36 & 2,70 & 0,00 & $-0,02$ & 5,8 & 2,6 & 1,7 \\
\hline Digitaria ciliaris & Poacée & 2 & 0,36 & 2,70 & 0,00 & $-0,02$ & 6,6 & 1,1 & 1,4 \\
\hline Hibiscus asper & Malvacée & 2 & 0,36 & 2,70 & 0,00 & $-0,02$ & 4,7 & 2,9 & 1,9 \\
\hline Nymphaea lotus & Nymphéacée & 2 & 0,36 & 2,70 & 0,00 & $-0,02$ & 4,4 & 1,0 & 17,5 \\
\hline Piliostigma reticulatum & Césalpinacée & 2 & 0,36 & 2,70 & 0,00 & $-0,02$ & 5,4 & 0,1 & 1,8 \\
\hline Sida rhombifolia & Malvacée & 2 & 0,36 & 2,70 & 0,00 & $-0,02$ & 6,2 & 2,4 & 4,6 \\
\hline
\end{tabular}


A. THIAM et al. / Int. J. Biol. Chem. Sci. 9(1): 155-175, 2015

\begin{tabular}{|c|c|c|c|c|c|c|c|c|c|}
\hline Alysicarpus ovalifolius & Papillonacée & 1 & 0,18 & 1,35 & 0,00 & $-0,01$ & 5,5 & 0,1 & 1,2 \\
\hline Andropogon gayanus & Poacée & 1 & 0,18 & 1,35 & 0,00 & $-0,01$ & 7,1 & 1,5 & 0,5 \\
\hline Chloris gayana & Poacée & 1 & 0,18 & 1,35 & 0,00 & $-0,01$ & 4,9 & 4,6 & 3,5 \\
\hline Conocarpus erectus & Combrétacée & 1 & 0,18 & 1,35 & 0,00 & $-0,01$ & 4,2 & 4,3 & 7,3 \\
\hline Crotolaria arenaria & Fabacée & 1 & 0,18 & 1,35 & 0,00 & $-0,01$ & 4,7 & 0,9 & 0,9 \\
\hline Indigofera tintoria & Papillonacée & 1 & 0,18 & 1,35 & 0,00 & $-0,01$ & 6,2 & 5,1 & 2,7 \\
\hline Ipomaea aquatica & Convolvulacée & 1 & 0,18 & 1,35 & 0,00 & $-0,01$ & 5,4 & 0,1 & 1,8 \\
\hline Kohautia grandiflora & Rubiacée & 1 & 0,18 & 1,35 & 0,00 & $-0,01$ & 4,3 & 1,3 & 3,2 \\
\hline Merremia tridentata & Convolvulacée & 1 & 0,18 & 1,35 & 0,00 & $-0,01$ & 6,6 & 0,4 & 0,2 \\
\hline Pakinsonia aculeata & Césalpinacée & 1 & 0,18 & 1,35 & 0,00 & $-0,01$ & 4,4 & 1,4 & 30,5 \\
\hline Pennisetum pedicellatum & Poacée & 1 & 0,18 & 1,35 & 0,00 & $-0,01$ & 5,4 & 0,1 & 1,8 \\
\hline Ziziphus mauritiana & Rhamnacée & 1 & 0,18 & 1,35 & 0,00 & $-0,01$ & 5,4 & 0,1 & 1,8 \\
\hline Total & & 551 & 100 & 744,594595 & 1 & $-3,2862904$ & & & \\
\hline
\end{tabular}


A. THIAM et al. / Int. J. Biol. Chem. Sci. 9(1): 155-175, 2015

Tableau 2 : Données floristiques du site de Ngan.

\begin{tabular}{|c|c|c|c|c|c|c|c|c|c|}
\hline Espèces & Familles & $\mathbf{P}$ & $\mathrm{CS} \%$ & FS\% & $\mathbf{P i}$ & $\mathbf{P i} * \mathbf{L n}(\mathbf{P i})$ & $\mathrm{Ce}$ & pH & Hum \\
\hline Cyperus rotundus & Cypéracée & 48 & 8,78 & 66,67 & 0,09 & $-0,21$ & 2,8 & 5,8 & 4,6 \\
\hline Ctenium elegans & Poacée & 47 & 8,59 & 65,28 & 0,09 & $-0,21$ & 2,8 & 5,8 & 4,6 \\
\hline Dactyloctenium aegyptium & Poacée & 47 & 8,59 & 65,28 & 0,09 & $-0,21$ & 2,8 & 5,7 & 4,6 \\
\hline Spermacoce verticillata & Rubiacée & 44 & 8,04 & 61,11 & 0,08 & $-0,20$ & 2,8 & 5,8 & 4,5 \\
\hline Enteropogon preurii & Poacée & 41 & 7,50 & 56,94 & 0,07 & $-0,19$ & 2,8 & 5,8 & 4,5 \\
\hline Philoxerus vermicularis & Amaranthacée & 38 & 6,95 & 52,78 & 0,07 & $-0,19$ & 2,3 & 5,8 & 4,6 \\
\hline Andropogon pseudapricus & Poacée & 28 & 5,12 & 38,89 & 0,05 & $-0,15$ & 3,3 & 5,3 & 5,6 \\
\hline Eragrostis squamata & Poacée & 28 & 5,12 & 38,89 & 0,05 & $-0,15$ & 1,1 & 5,6 & 2,5 \\
\hline Spermacoce stachydea & Rubiacée & 23 & 4,20 & 31,94 & 0,04 & $-0,13$ & 2,8 & 5,6 & 4,6 \\
\hline Zornia glochidiata & Fabacée & 21 & 3,84 & 29,17 & 0,04 & $-0,13$ & 2,7 & 5,2 & 4,6 \\
\hline Balanites aegyptiaca & Simaroubacée & 18 & 3,29 & 25,00 & 0,03 & $-0,11$ & 2,8 & 5,6 & 4,6 \\
\hline Cassia obstusifolia & Césalpinacée & 15 & 2,74 & 20,83 & 0,03 & $-0,10$ & 3,3 & 5,7 & 5,7 \\
\hline Eragrostis tenella & Poacée & 15 & 2,74 & 20,83 & 0,03 & $-0,10$ & 2,3 & 5,5 & 4,6 \\
\hline Kohautia grandiflora & Rubiacée & 14 & 2,56 & 19,44 & 0,03 & $-0,09$ & 0,8 & 6,2 & 2,3 \\
\hline Eragrostis tremula & Poacée & 13 & 2,38 & 18,06 & 0,02 & $-0,09$ & 0,8 & 6,1 & 1,2 \\
\hline Peristrophe bicalyculata & Acanthacée & 10 & 1,83 & 13,89 & 0,02 & $-0,07$ & 3,3 & 5,5 & 0,3 \\
\hline Acacia seyal & Mimosacée & 8 & 1,46 & 11,11 & 0,01 & $-0,06$ & 3,3 & 5,6 & 5,0 \\
\hline Barchiaria ramosa & Poacée & 8 & 1,46 & 11,11 & 0,01 & $-0,06$ & 1,0 & 5,3 & 2,5 \\
\hline Acacia nilotica & Mimosacée & 7 & 1,28 & 9,72 & 0,01 & $-0,06$ & 2,8 & 5,2 & 4,7 \\
\hline Commelina forskalaei & Commelinacée & 7 & 1,28 & 9,72 & 0,01 & $-0,06$ & 0,8 & 6,3 & 1,2 \\
\hline Corchorus olitorius & Tiliacée & 6 & 1,10 & 8,33 & 0,01 & $-0,05$ & 1,2 & 5,0 & 0,9 \\
\hline Sida rhombifolia & Malvacée & 6 & 1,10 & 8,33 & 0,01 & $-0,05$ & 1,7 & 5,3 & 2,8 \\
\hline Chloris pilosa & Poacée & 5 & 0,91 & 6,94 & 0,01 & $-0,04$ & 0,8 & 6,8 & 1,2 \\
\hline
\end{tabular}


A. THIAM et al. / Int. J. Biol. Chem. Sci. 9(1): 155-175, 2015

\begin{tabular}{|c|c|c|c|c|c|c|c|c|c|}
\hline Ipomaea asarifolia & Convolvulacée & 5 & 0,91 & 6,94 & 0,01 & $-0,04$ & 1,2 & 5,7 & 0,9 \\
\hline Schoenefeldia gracilis & Poacée & 5 & 0,91 & 6,94 & 0,01 & $-0,04$ & 0,0 & 6,7 & 0,2 \\
\hline Sphaeranthus senegalensis & Astéracée & 5 & 0,91 & 6,94 & 0,01 & $-0,04$ & 3,4 & 6,1 & 2,2 \\
\hline Thypha australis & Poacée & 5 & 0,91 & 6,94 & 0,01 & $-0,04$ & 1,0 & 5,3 & 2,8 \\
\hline Calotropis procera & Asclépiadacée & 4 & 0,73 & 5,56 & 0,01 & $-0,04$ & 0,0 & 6,2 & 0,1 \\
\hline Ampelocisus pentaphylla & Vitacée & 3 & 0,55 & 4,17 & 0,01 & $-0,03$ & 2,3 & 5,3 & 1,5 \\
\hline Scoparia dulcis & Scrophulariacée & 3 & 0,55 & 4,17 & 0,01 & $-0,03$ & 0,0 & 5,8 & 0,4 \\
\hline Waltheria indica & Tiliacée & 3 & 0,55 & 4,17 & 0,01 & $-0,03$ & 1,2 & 5,6 & 0,9 \\
\hline Anarcadium occidentalis & Anarcadiacée & 2 & 0,37 & 2,78 & 0,00 & $-0,02$ & 0,0 & 5,4 & 0,6 \\
\hline Cenchrus biflorus & Poacée & 2 & 0,37 & 2,78 & 0,00 & $-0,02$ & 1,5 & 6,9 & 0,5 \\
\hline Crotalaria glaucoïdes & Fabacée & 2 & 0,37 & 2,78 & 0,00 & $-0,02$ & 0,0 & 5,4 & 0,6 \\
\hline Hibiscus asper & Malvacée & 2 & 0,37 & 2,78 & 0,00 & $-0,02$ & 0,2 & 4,5 & 0,1 \\
\hline Ocimum basilicum & Lamiacée & 2 & 0,37 & 2,78 & 0,00 & $-0,02$ & 0,0 & 5,9 & 1,0 \\
\hline Pennisetum pedicellatum & Poacée & 2 & 0,37 & 2,78 & 0,00 & $-0,02$ & 0,0 & 5,4 & 0,6 \\
\hline Sesuvium portulacastrum & Aizoacée & 2 & 0,37 & 2,78 & 0,00 & $-0,02$ & 5,5 & 4,5 & 9,0 \\
\hline Acacia albida & Mimosacée & 1 & 0,18 & 1,39 & 0,00 & $-0,01$ & 1,7 & 6,0 & 1,0 \\
\hline Cassia nigricans & Césalpinacée & 1 & 0,18 & 1,39 & 0,00 & $-0,01$ & 0,0 & 5,5 & 0,9 \\
\hline Cynodon dactylon & Poacée & 1 & 0,18 & 1,39 & 0,00 & $-0,01$ & 1,7 & 6,0 & 1,0 \\
\hline Total & & 547 & 100,00 & & & $-3,19$ & & & \\
\hline
\end{tabular}

$F=$ Famille, $P=$ Présence, $C S \%=$ Contribution spécifique en $\% F S=$ Fréquence spécifique en $\%$ P $i=$ Richesse spécifique.

$C e=$ Conductivité électrique $(d S / m) . p H=$ Potentiel hydrogène. Hum = Taux d'humidité 


\section{DISCUSSION}

Dans le cadre de notre étude les résultats obtenus sur la distribution des tannes au niveau des unités géomorphologiques ont montré que la majorité des tannes sont observées sur la terrasse moyenne avec 50\% au nord du fleuve Saloum (Ngan) et $40 \%$ au sud du fleuve Saloum (Ndiaffate). Ce résultat confirme les travaux de Sadio (1986) qui a noté qu'au niveau de la terrasse moyenne on y rencontre une diversification des types de tannes par rapport aux autres unités géomorphologiques.

Pour l'étude de la végétation en fonction des unités géomorphologiques et des types de faciès, nous avons constaté sur sols à texture fine (taux d'argile > 24\%, sud du fleuve Saloum) que la diversité biologique est plus importante par rapport au sol dont la texture est grossière (taux de sable de 55\%, nord du fleuve Saloum). Dans le secteur de Ndiaffate, 49 espèces ont été recensées contre 41 dans le secteur de Ngan. Ces espèces sont réparties en classes en fonction de leur fréquence et de leur contribution spécifique.

Ces résultats sont en parfaite adéquation d'une part avec ceux des travaux de plusieurs auteurs (Bille, 1977 ; Cornet, 1981 ; Barral et al. 1983) qui ont mis en évidence les variations de la structure spécifique de la végétation en relation avec les conditions édapho-climatiques, et d'autre part avec ceux de Thiaw et al. (2011) dans la caractérisation de la végétation herbacée dans la région de Kaffrine (Centre Ouest Sénégal). Ils ont mis en évidence le regroupement de certaines espèces en fonction des paramètres physico-chimique des sols à savoir le regroupement à Téphrosia pedicelleta sur sol à texture argilo-limoneuse et le groupement Ipomoea pestigridis et Ipomoea vagans sur sols à texture sableuse. Ils ont mis en évidence la richesse floristique de cette zone avec 63 espèces herbacées réparties dans 50 genres et 20 familles.

Les résultats obtenus sur la répartition en classe en fonction de leur fréquence et de leur contribution spécifique sont en conformité avec celles observées par Ashraf (2008) pour la distribution de la végétation en fonction des facteurs édaphiques dans le désert du Cholistan (Pakistan). Les travaux de Diallo et al. (2009) sur la variabilité de la végétation herbacée des Niayes de Pikine sont en parfaite adéquation avec nos résultats obtenus dans le bassin arachidier. Dans cette étude (Diallo et al., 2009), cette forte variabilité floristique riche de 43 familles représentées par 119 genres et 163 espèces est un témoin de l'impact de la sécheresse, de l'augmentation de la salinité et l'action anthropique. Il apparaît clairement dans cette étude que les différents types de végétation correspondent aux niveaux de salinité et d'humidité du sol, de la matière organique et de la concentration ionique.

Les travaux de Jafari et al. (2004) sur les relations végétation - sol dans la région de Hoz-e-Soltan (Iran) ont révélé l'existence d'une relation spécifique entre les caractéristiques du sol et la distribution de la végétation. Les caractéristiques du sol étaient la salinité et la texture. Dans notre étude, nous avons ciblé la texture, l'humidité, la salinité et le $\mathrm{pH}$ comme variables. Notre démarche est, en partie, en adéquation avec celle de Kassas (1957), Ungar (1968), Jafari (1989), Moghimi (1989), Caballero et al., (1994), Maryam et al. (1995) et WEI-Qiang et al. (2008) sur les relations entre les caractéristiques des sols et la végétation halophyte.

Ces auteurs ont constaté que la distribution de la végétation dans une région donnée est fonction de la salinité du sol. AbuZiada (1980) a pu démontrer une forte relation de la distribution de la végétation avec la salinité et l'humidité dans le sol. Les travaux 
de Jafari et al. (2004), comme précédemment, ont indiqué que la texture du sol est une variable a un effet sur la distribution de la végétation car elle a une influence sur le taux et les substances nutritives disponibles dans le sol. Dans notre étude, l'utilisation de l'ACP avec comme variable effective la texture, a permis la mise en évidence à Ndiaffate comme à Ngan des groupes d'espèces de tailles différentes pour chaque texture: sableuse, sablo-limoneuse et limono-sableuse. Cela nous a permis de distinguer des espèces végétales spécifiques colonisant des types de texture de sols donnés et sur des gammes spécifiques de salinité, d'humidité et d'acidité.

Il apparaît clairement au niveau du Sud du fleuve Saloum que les espèces rencontrées strictement sur sols à texture sableuse, sablolimoneuse et limono-sableuse caractérisent mieux ces milieux. Lorsque la texture est sableuse, Chloris pilosa et Merremia tridentata sont les mieux indiquées. Elles caractérisent les sols légèrement salins à salins avec une acidité variant de l'acide au faiblement acide avec des teneurs d'humidité variant $0,23 \%$ à $5 \%$.

Lorsque la texture du sol est sablo limoneuse, les espèces Conocarpus erectus, Balanites aegyptiaca et Kohautia grandifolia sont les mieux adaptées pour faire la caractérisation de ces sols sulfatés acides salés. Elles indiquent les milieux caractérisés par une teneur en humidité de $1,25 \%$ à $7,25 \%$, avec des caractères de salinité de moyennement salin à très salin et un caractère d'acidité évoluant du très acide au faiblement acide. Les espèces enregistrées strictement lorsque la texture est limono-sableuse dont les pourcentages de contribution sont les plus élevés sont: Pakinsonia aculeata, Andropogon gayanus et Nymphaea lotus. Ces espèces caractérisent ces sols dont les caractères de salinité évoluent du non salins à salins avec un caractère d'acidité allant du très acide à neutre pour une humidité de $0,51 \%$ à $30,51 \%$.

Au nord du fleuve Saloum, les espèces Hibiscus asper et Calotropis procera sont rencontrées uniquement sur sols à texture sableuse. Ces espèces indiquent un milieu sableux avec des caractères non salins à légèrement salins. Les caractères d'acidité enregistrés varient de l'acide au faiblement acide. L'humidité varie entre 0,07 et $1,03 \%$.

L'espèce rencontrée strictement sur sols sulfatés acides salés à texture sablolimoneuse est Sesuvium portulacastrum. Elle indique un milieu salin avec un caractère acide et un taux d'humidité de $9 \%$. Les espèces strictement rencontrées lorsqu'on a une texture limono-sableuse sont: Kohautia grandiflora, Anarcadium occidentalis, Crotalaria glaucoüdes et Pennisetum pedicellatum. Ces espèces indiquent un milieu dont la gamme de salinité varie entre non salin et moyennement salin avec un caractère très acide à faiblement acide pour une humidité du sol variant entre 0,38 et $1,78 \%$. Ces résultats confirment ceux de Jafari et al., (2004), qui sont parvenus à identifier des espèces indicatrices des sols de salinité faible et de texture grossière (Artemisia sieberi) et des sols à salinité moyenne à forte (Tamarix sp., Suaeda aegyptiaca).

Dans notre étude, nous avons tenu compte des recommandations faites par les auteurs cités plus haut pour une classification des espèces végétales dans les sols (Ndiaffate et Ngan) en fonction des types de textures et des gammes maximales et minimales du taux d'humidité, du caractère d'acidité et de salinité. Ces derniers ont observé l'influence de la texture du sol sur la distribution de la végétation. Cette texture affecte le taux d'humidité du sol et par conséquent la nutrition des espèces affectant ainsi leur distribution. 


\section{Conclusion}

L'étude de la végétation en fonction des unités géomorphologiques et du type de faciès montre que la distribution du type de tannes, du nombre d'espèces et de genres n'est pas fonction du type de terrasses au niveau des deux sites et même à l'intérieur d'un même site. Cependant malgré les différences pluviométriques et les types de sols dans les deux secteurs, la salinité et l'acidité sont en grande partie responsables de cette similarité végétale entre les deux secteurs et à l'intérieur d'un même secteur. La distribution aires/espèces, à travers les différentes grappes dans les secteurs d'études, a révélé que la déclivité du terrain n'est pas le seul facteur explicatif de ce façonnement du faciès écologique (type de tannes).

La distribution spatio-temporelle très hétérogène des éléments de contraintes (salinité et acidité) permet de mettre en évidence la diversité des faciès écologiques à l'intérieur d'une même unité géomorphologique ou entre deux unités géomorphologiques différentes se succédant. Dans ces milieux, les familles les plus représentées sont les Poacées et les Rubiacées. La distribution des espèces en fonction des éléments de contraintes s'appuie sur les propriétés du sol.

La connaissance du mode de regroupement de la végétation en fonction des paramètres de contraintes (salinité et acidité) et de la texture des sols est susceptible de donner d'une part, des informations sur le choix du type d'aménagement, à savoir les dispositifs biomécaniques et hydro agricoles tout en mettant l'accent sur le drainage des sols pour éviter une salinisation secondaire. D'autre part, elle aiderait sur la caractérisation de ces milieux par la réduction des coûts d'analyses des échantillons de sols, l'élaboration d'une carte des textures de sol, la mise en évidence de la complexité de la couverture pédologique et de la diversité des types de sols surtout à l'échelle parcellaire, l'enchevêtrement de ces sols dans l'espace ainsi que la coexistence de l'acidité et la salinité.

\section{REFERENCES}

Abu-Ziada MEA. 1980. Ecological studies on the flora of Kharga and Dakhla Oases of the Western Desert of Egypt. Ph.D Thesis, Fac. Sci., Mansoura Univ., 342 pp.

Arshrad M. 2003. Cholistan desert ecosystem monitoring for future management. Annual Technical Report of a project sponsored by WWF-Pakistan. (Unpublished).

Battistini R. 1960. Description géomorphologique de Nosy-Be, du delta du Sambirano et de la baie d'Ampasindava. Mémoires de l'Institut des Sciences de Madagascar, série F, tome 3, 121-343.

Berthois L, Guilcher A. 1956. Questions de morphologie climatique en Mélanésie équatoriale (Novelle Guinée, Nouvelle Bretagne, Guadalcamal). Bull. ASS. Géographes Fr, 338-339.

Braun-Blanquet 1928. Pflanzensoziologie. Grundzüge der Vegetationskunde. Biol. Studienbücher, 7, Berlin. In Elément de Biogéographie et d'Ecologie, Lacoste A, Salanon R (eds). Nathan: Paris; 189.

Caballero JM, Esteve MA, Calvo JF, Pujol JA. 1994. Structure of the vegetation of salt steppes of Guadelenitin (Murcia, Spain). Stud. Oecol., 10(11): 171-183.

Diallo A, Guissé A, Faye Ngom M, Saradoum G. 2009. Variabilité floristique de la végétation herbacée de la niaye de Pikine au Sénégal. Rev. Écol. (Terre Vie), 64: 123-133.

Fleur TJ. 1975. Halophytes. In Ion Transport in Cells and Tissues, Barker DA, Hall JL 
(eds). North Holland, Amesterdam; 309334.

Jafari M. 1989. Survey of relationship between salinity agents and distribution of plants in Damghan region. MSc. thesis in Natural Resources College of Tarbiat Modarres university (Persian)

Jafari M, Chahouki MAZ, Tavili A, Azarnivand H, Amiri GZ. 2004. Effective environmental factors in the distribution of vegetation types in Poshtkouh rangelands of Yazd Province (Iran). Journal of Arid Environments, 56: 627-641.

Kassas M. 1957. On the ecology of the Red Sea coastal land. J. Ecolo., 45: 187-203.

Lebigre M. 1984. Les mangroves des rias du littoral Gabonais, essai de cartographie et typologie. Bois et Forêts Tropicaux, 199: 3-28.

Marius C. 1985. Mangroves du Sénégal et de la Gambie. Ecologie-PédologieGéochimie. Mise en valeur et aménagement. Mémoire ORSTOM $\mathrm{n}^{\circ} 106$, Paris.

Maryam H, Ismail S, Alaa F, Ahmed R. 1995. Studies on growth and salt regulation in some halophytes as influenced by edaphic and climatic conditions. Pak. J. Bot., 27: 151-163.

Moghimi J. 1989. Survey of relationship between vegetation, salinity and water ground table in Hoz-e- Soltan playa in Qom province, MSc. thesis in Natural Resources College of Tehran university (Persian).

Muhammad YA. 2008. Impact of salt stress on concentration of $\mathrm{Na}^{+}, \mathrm{Cl}^{-}$, and organic solutes concentration in pea cultivars.
Pansu M, Gautheyron J. 2003. L'Analyse du Sol, Minéralogie, Organique et Minérale. Ed Springer-verlag. France, $993 \mathrm{p}$

Sadio S. 1986. Etude pédologique des facteurs de mortalité des essences forestières introduites en milieux salés au Sénégal. ORSTOM/Dakar, 66 p + 34 p. an.

Thiam A. 2007. Rapport d'activité. Caractérisation de la végétation sur sols sulfatés acides salés dans la zone de Ngane Kaolack ISRA (Centre de Kaolack). 12 p.

Thiam. A. 2008. Rapport des travaux d'avancement : Caractérisation du milieu physique des sites expérimentaux (Ndiaffate et Ngane) dans le domaine des tannes, Kaolack ISRA (Centre de Kaolack), 35 p.

Thiaw A, Faye MN, Diallo A, Ndiaye O, Fall RD, Guissé A. 2011 : Caractérisation de la végétation herbacée de la région de Kaffrine (Centre-Ouest, Sénégal). Annales des Sciences Agronomiques, 15(2): 243-264.

Ungar I. 1968. Species-soil relationships on the Great Salt Plains of northern Oklahoma. Amer. Midl. Naturalist, 80: 392-406.

WEI-Qiang Li, Liu Xiao-Jing, Ajmal Khan M, Bilquees Gul. 2008. Relationship between soil characteristics and halophytic vegetation in coastal region of North China. Pak. J. Bot., 40(3): 1081-1090. 